\title{
Interpolation Problem for Multidimensional Stationary Processes with Missing Observations
}

\author{
Oleksandr Masyutka ${ }^{1}$, Mikhail Moklyachuk $^{2, *}$, Maria Sidei $^{2}$ \\ ${ }^{1}$ Department of Mathematics and Theoretical Radiophysics, Taras Shevchenko National University of Kyiv,Ukraine \\ ${ }^{2}$ Department of Probability Theory, Statistics and Actuarial Mathematics, Taras Shevchenko National University of Kyiv,Ukraine
}

\begin{abstract}
The problem of the mean-square optimal linear estimation of linear functionals which depend on the unknown values of a multidimensional continuous time stationary stochastic process is considered. Estimates are based on observations of the process with an additive stationary stochastic noise process at points which do not belong to some finite intervals of a real line. The problem is investigated in the case of spectral certainty, where the spectral densities of the processes are exactly known. Formulas for calculating the mean-square errors and spectral characteristics of the optimal linear estimates of functionals are proposed under the condition of spectral certainty. The minimax (robust) method of estimation is applied in the case of spectral uncertainty, where spectral densities of the processes are not known exactly while some sets of admissible spectral densities of the processes are given. Formulas that determine the least favorable spectral densities and the minimax spectral characteristics of the optimal estimates of functionals are proposed for some special sets of admissible spectral densities.
\end{abstract}

Keywords Stationary process, optimal estimate, mean square error, minimax-robust estimate, least favorable spectral density, minimax spectral characteristic

AMS 2010 subject classifications. Primary: 60G10, 60G25, 60G35, Secondary: 62M20, 93E10, 93E11

DOI: $10.19139 /$ soic.v7i1.430

\section{Introduction}

The problem of estimation of the unknown values of stochastic processes is of constant interest in the theory and applications of stochastic processes. The description of the problem and effective methods of solution of the interpolation, extrapolation and filtering problems for stationary stochastic sequences and processes were were developed by Kolmogorov [17], Wiener [40] and Yaglom [41, 42]. Further development of the methods of solution and analysis of the problems can be found in the works by Rozanov [37] and Hannan [12], Box et. al [3], Brockwell and Davis [4]. The basic assumption of most of the methods of solution of the problem of estimation of the unobserved values of stochastic processes is that the spectral densities of the considered stochastic processes are exactly known. However, in practice these methods can not be applied since the complete information on the spectral densities is impossible in most cases. In order to solve the problem, the parametric or nonparametric estimates of the unknown spectral densities are found. Then, one of the traditional estimation methods is applied, provided that the selected spectral densities are true. This procedure can result in significant increase in the value of the error of estimate as Vastola and Poor [39] have demonstrated with the help of some examples. To avoid this effect one can search estimates which are optimal for all densities from a certain class of admissible spectral densities. These estimates are called minimax since they minimize the maximum value of *Correspondence to: Mikhail Moklyachuk (Email: Moklyachuk@ gmail.com). Department of Probability Theory, Statistics and Actuarial
Mathematics, Taras Shevchenko National University of Kyiv, Volodymyrska 64 Str., Kyiv 01601, Ukraine.

ISSN 2310-5070 (online) ISSN 2311-004X (print)

Copyright (C) 2019 International Academic Press 
the error. The paper by Grenander [11] should be marked as the first one where this approach to extrapolation problem for stationary processes was proposed. Several models of spectral uncertainty and the minimax-robust methods of data processing can be found in the survey paper by Kassam and Poor [16]. In their papers, Franke [7, 8], Franke and Poor [9] presented results of the investigation of the minimax extrapolation and filtering problems for stationary sequences with the help of convex optimization methods. This approach makes it possible to find relations that determine the least favorable spectral densities for different classes of admissible spectral densities. The papers by Moklyachuk [23,24] is dedicated to the investigation of the problems of the optimal linear estimation of functionals which depend on the unknown values of stationary sequences and processes. The problem of estimation of functionals which depend on the unknown values of multivariate stationary stochastic processes is the aim of the papers by Moklyachuk and Masyutka [26] - [28]. In the book by Moklyachuk and Golichenko [25] results of investigation of the interpolation, extrapolation and filtering problems for periodically correlated stochastic processes are described. Estimation problems for functionals which depend on the unknown values of the stochastic processes with stationary increments were investigated by Luz and Moklyachuk [18][21]. The problem of interpolation, extrapolation and filtering for stationary processes with missing observations were investigated by Moklyachuk and Sidei [29] - [32]. The prediction and interpolation problems for stationary stochastic sequences with missing observations were investigated in papers by Bondon [1, 2], Cheng, Miamee and Pourahmadi [5], Cheng and Pourahmadi [6], Kasahara, Pourahmadi and Inoue [15], Pourahmadi, Inoue and Kasahara [34], Pelagatti [33]. The problem of interpolation of stationary sequences was investigated in the paper of Salehi [38].

In this paper we deal with the problem of the mean-square optimal linear estimation of the functional

$$
A_{s} \vec{\xi}=\sum_{l=1}^{s} \int_{-M_{l}-N_{l}}^{-M_{l}} \vec{a}(t)^{\top} \vec{\xi}(t) d t
$$

which depends on the unknown values of a multidimensional continuous time stationary stochastic process $\vec{\xi}(t)$ from observations of the process with an additive stationary stochastic noise process $\vec{\xi}(t)+\vec{\eta}(t)$ at points $t \in \mathbb{R} \backslash S$, $S=\bigcup_{l=1}^{s}\left[-M_{l}-N_{l},-M_{l}\right]$. The case of spectral certainty as well as the case of spectral uncertainty are considered. In the case of spectral certainty, where the spectral densities of the processes are exactly known, formulas for calculating the spectral characteristic and the mean square error of the optimal linear estimate of the functional are derived. In the case of spectral uncertainty, where the spectral densities are not exactly known while a set of admissible spectral densities is given, the minimax method is applied. This method allows us to find estimates that minimize the maximum values of the mean-square errors of estimates for all spectral density matrices from a given class of admissible spectral density matrices. Formulas that determine the least favorable spectral densities and the minimax-robust spectral characteristics of the optimal estimates of the functional are proposed for some specific classes of admissible spectral densities.

\section{Hilbert space projection approach to interpolation problem for stationary processes with missing observations}

Let $\vec{\xi}(t)=\left\{\xi_{k}(t)\right\}_{k=1}^{T}, t \in \mathbb{R}$, and $\vec{\eta}(t)=\left\{\eta_{k}(t)\right\}_{k=1}^{T}, t \in \mathbb{R}$, be uncorrelated mean square continuous multidimensional stationary stochastic processes with zero first moments, $E \vec{\xi}(t)=\overrightarrow{0}, E \vec{\eta}(t)=\overrightarrow{0}$, and correlation functions which admit the spectral decomposition (see Gikhman and Skorokhod [10])

$$
R_{\xi}(t)=\frac{1}{2 \pi} \int_{-\infty}^{\infty} e^{i t \lambda} F(\lambda) d \lambda, \quad R_{\eta}(t)=\frac{1}{2 \pi} \int_{-\infty}^{\infty} e^{i t \lambda} G(\lambda) d \lambda
$$


where $F(\lambda)=\left\{f_{k l}(\lambda)\right\}_{k, l=1}^{T}$ and $G(\lambda)=\left\{g_{k l}(\lambda)\right\}_{k, l=1}^{T}$ are spectral density matrices of the processes $\vec{\xi}(t)$ and $\vec{\eta}(t)$ respectively which satisfy the minimality condition

$$
\int_{-\infty}^{\infty}(b(\lambda))^{\top}(F(\lambda)+G(\lambda))^{-1} \overline{b(\lambda)} d \lambda<\infty
$$

where $b(\lambda)=\sum_{l=1}^{s} \int_{-M_{l}-N_{l}}^{-M_{l}} \vec{\alpha}(t) e^{i t \lambda} d t$ is a nontrivial function of the exponential type. Under this condition the errorfree interpolation of the process $\vec{\xi}(t)+\vec{\eta}(t)$ is impossible (see Rozanov [37]).

The stationary processes $\vec{\xi}(t)$ and $\vec{\eta}(t)$ admit the spectral decompositions (see Gikhman and Skorokhod [10], Karhunen [14])

$$
\vec{\xi}(t)=\int_{-\infty}^{\infty} e^{i t \lambda} Z_{\xi}(d \lambda), \quad \vec{\eta}(t)=\int_{-\infty}^{\infty} e^{i t \lambda} Z_{\eta}(d \lambda),
$$

where $Z_{\xi}(d \lambda)$ and $Z_{\eta}(d \lambda)$ are vector valued orthogonal stochastic measures such that

$$
\begin{aligned}
& E Z_{\xi}\left(\Delta_{1}\right)\left(Z_{\xi}\left(\Delta_{2}\right)\right)^{*}=\frac{1}{2 \pi} \int_{\Delta_{1} \cap \Delta_{2}} F(\lambda) d \lambda, \\
& E Z_{\eta}\left(\Delta_{1}\right)\left(Z_{\eta}\left(\Delta_{2}\right)\right)^{*}=\frac{1}{2 \pi} \int_{\Delta_{1} \cap \Delta_{2}} G(\lambda) d \lambda .
\end{aligned}
$$

Consider the problem of the mean-square optimal linear estimation of the functional

$$
A_{s} \vec{\xi}=\sum_{l=1}^{s} \int_{-M_{l}-N_{l}}^{-M_{l}} \vec{a}(t)^{\top} \vec{\xi}(t) d t
$$

which depends on the unknown values of the process $\vec{\xi}(t)$. Estimates are based on observations of the process $\vec{\xi}(t)+\vec{\eta}(t)$ at points $t \in \mathbb{R} \backslash S$, where $S=\bigcup_{l=1}^{s}\left[-M_{l}-N_{l},-M_{l}\right]$.

We will assume that the function $\vec{a}(t)$ satisfies the following conditions

$$
\sum_{k=1}^{T} \sum_{l=1}^{s} \int_{-M_{l}-N_{l}}^{-M_{l}}\left|a_{k}(t)\right| d t<\infty
$$

This condition ensures that the functional $A_{s} \vec{\xi}$ has a finite second moment.

Making use of the spectral decomposition (2) of the stationary process $\vec{\xi}(t)$, we can represent the functional $A_{s} \vec{\xi}$ in the following form

$$
A_{s} \vec{\xi}=\int_{-\infty}^{\infty}\left(A_{s}(\lambda)\right)^{\top} Z_{\xi}(d \lambda), \quad A_{s}(\lambda)=\sum_{l=1}^{s} \int_{-M_{l}-N_{l}}^{-M_{l}} \vec{a}(t) e^{i t \lambda} d t
$$

Denote by $\hat{A}_{s} \vec{\xi}$ the optimal linear estimate of the functional $A_{s} \vec{\xi}$ from observations of the process $\vec{\xi}(t)+\vec{\eta}(t)$ and denote by $\Delta(F, G)=E\left|A_{s} \vec{\xi}-\hat{A}_{s} \vec{\xi}\right|^{2}$ the mean-square error of the estimate $\hat{A}_{s} \vec{\xi}$. Since the spectral densities of the stationary processes $\vec{\xi}(t)$ and $\vec{\eta}(t)$ are suppose to be known, we can use the method of orthogonal projections in Hilbert spaces proposed by Kolmogorov [17] to find the estimate $\hat{A}_{s} \vec{\xi}$. 
Consider values $\xi_{k}(t), k=1, \ldots, T, t \in \mathbb{R}$ and $\eta_{k}(t), k=1, \ldots, T, t \in \mathbb{R}$ as elements of the Hilbert space $H=L_{2}(\Omega, \mathcal{F}, P)$ generated by random variables $\xi$ with zero mathematical expectations, $E \xi=0$, finite variations, $E|\xi|^{2}<\infty$, and inner product $(\xi, \eta)=E \xi \bar{\eta}$. Denote by $H^{s}(\xi+\eta)$ the closed linear subspace generated by elements $\left\{\xi_{k}(t)+\eta_{k}(t): t \in \mathbb{R} \backslash S, k=\overline{1, T}\right\}$ in the Hilbert space $H=L_{2}(\Omega, \mathcal{F}, P)$.

Denote by $L_{2}(F+G)$ the Hilbert space of vector-valued functions $\vec{a}(\lambda)=\left\{a_{k}(\lambda)\right\}_{k=1}^{T}$ such that

$$
\int_{-\infty}^{\infty} \vec{a}(\lambda)^{\top}(F(\lambda)+G(\lambda)) \overline{\vec{a}(\lambda)} d \lambda=\int_{-\infty}^{\infty} \sum_{k, l=1}^{T} a_{k}(\lambda) \overline{a_{l}(\lambda)}\left(f_{k l}(\lambda)+g_{k l}(\lambda)\right) d \lambda<\infty,
$$

and denote by $L_{2}^{s}(F+G)$ the subspace of the space $L_{2}(F+G)$ generated by the functions $\left\{e^{i t \lambda} \delta_{k}, \delta_{k}=\right.$ $\left.\left\{\delta_{k l}\right\}_{l=1}^{T}, k=\overline{1, T}, t \in \mathbb{R} \backslash S\right\}$.

The mean-square optimal linear estimate $\hat{A}_{s} \vec{\xi}$ of the functional $A_{s} \vec{\xi}$ can be represented in the form

$$
\hat{A}_{s} \vec{\xi}=\int_{-\infty}^{\infty}(h(\lambda))^{\top}\left(Z_{\xi}(d \lambda)+Z_{\eta}(d \lambda)\right)
$$

where $h(\lambda) \in L_{2}^{s}(F+G)$ is the spectral characteristic of the estimate.

The mean-square error $\Delta(h ; F, G)$ of the estimate $\hat{A}_{s} \vec{\xi}$ is given by the formula

$$
\begin{gathered}
\Delta(h ; F, G)=E\left|A_{s} \xi-\hat{A}_{s} \xi\right|^{2}= \\
=\frac{1}{2 \pi} \int_{-\infty}^{\infty}\left(A_{s}(\lambda)-h(\lambda)\right)^{\top} F(\lambda) \overline{\left(A_{s}(\lambda)-h(\lambda)\right)} d \lambda+\frac{1}{2 \pi} \int_{-\infty}^{\infty}(h(\lambda))^{\top} G(\lambda) \overline{h(\lambda)} d \lambda .
\end{gathered}
$$

According to the Hilbert space projection method the optimal estimate of the functional $A_{s} \vec{\xi}$ is a projection of the element $A_{s} \vec{\xi}$ of the space $H$ on the subspace $H^{s}(\xi+\eta)$. It can be found from the following conditions:

$$
\begin{array}{ll}
\text { 1) } & \hat{A}_{s} \vec{\xi} \in H^{s}(\xi+\eta), \\
\text { 2) } & A_{s} \vec{\xi}-\hat{A}_{s} \vec{\xi} \perp H^{s}(\xi+\eta) .
\end{array}
$$

It follows from the second condition that the spectral characteristic $h(\lambda)=\left\{h_{k}(\lambda)\right\}_{k=1}^{T}$ of the optimal linear estimate $\hat{A}_{s} \vec{\xi}$ for any $t \in \mathbb{R} \backslash S$ satisfies the equation

$$
\frac{1}{2 \pi} \int_{-\infty}^{\infty}\left(A_{s}(\lambda)-h(\lambda)\right)^{\top} F(\lambda) e^{-i t \lambda} d \lambda-\frac{1}{2 \pi} \int_{-\infty}^{\infty}(h(\lambda))^{\top} G(\lambda) e^{-i t \lambda} d \lambda=0 .
$$

The last relation is equivalent to equations

$$
\frac{1}{2 \pi} \int_{-\infty}^{\infty}\left[\left(A_{s}(\lambda)\right)^{\top} F(\lambda)-(h(\lambda))^{\top}(F(\lambda)+G(\lambda))\right] e^{-i t \lambda} d \lambda=0, \quad t \in \mathbb{R} \backslash S .
$$

Define the function

$$
\left(C_{s}(\lambda)\right)^{\top}=\left(A_{s}(\lambda)\right)^{\top} F(\lambda)-(h(\lambda))^{\top}(F(\lambda)+G(\lambda))
$$

and its Fourier transform

$$
\vec{c}(t)=\frac{1}{2 \pi} \int_{-\infty}^{\infty} C_{s}(\lambda) e^{-i t \lambda} d \lambda, \quad t \in \mathbb{R}
$$


It follows from the relation (4) that the function $\vec{c}(t)$ is nonzero only on the set $S$. Hence, the function $C_{s}(\lambda)$ is of the form

$$
C_{s}(\lambda)=\sum_{l=1}^{s} \int_{-M_{l}-N_{l}}^{-M_{l}} \vec{c}(t) e^{i t \lambda} d t
$$

and the spectral characteristic of the estimate $\hat{A}_{s} \vec{\xi}$ is of the form

$$
(h(\lambda))^{\top}=\left(A_{s}(\lambda)\right)^{\top} F(\lambda)(F(\lambda)+G(\lambda))^{-1}-\left(C_{s}(\lambda)\right)^{\top}(F(\lambda)+G(\lambda))^{-1} .
$$

It follows from the first condition, $\hat{A}_{s} \vec{\xi} \in H^{s}(\xi+\eta)$, which determine the optimal linear estimate of the functional $A_{s} \vec{\xi}$, that the following relation holds true

$$
\int_{-\infty}^{\infty}\left(\left(A_{s}(\lambda)\right)^{\top} F(\lambda)(F(\lambda)+G(\lambda))^{-1}-\left(C_{s}(\lambda)\right)^{\top}(F(\lambda)+G(\lambda))^{-1}\right) e^{-i t \lambda} d \lambda=0, t \in S .
$$

The last relation can be represented in terms of linear operators in the space $L_{2}(S)$. Let us define operators

$$
\begin{aligned}
\left(\mathbf{B}_{s} \mathbf{a}\right)(t) & =\frac{1}{2 \pi} \sum_{l=1}^{s} \int_{-M_{l}-N_{l}}^{-M_{l}} \int_{-\infty}^{\infty}(\vec{a}(u))^{\top}(F(\lambda)+G(\lambda))^{-1} e^{i \lambda(u-t)} d \lambda d u, \\
\left(\mathbf{R}_{s} \mathbf{a}\right)(t) & =\frac{1}{2 \pi} \sum_{l=1}^{s} \int_{-M_{l}-N_{l}}^{-M_{l}} \int_{-\infty}^{\infty}(\vec{a}(u))^{\top} F(\lambda)(F(\lambda)+G(\lambda))^{-1} e^{i \lambda(u-t)} d \lambda d u \\
\left(\mathbf{Q}_{s} \mathbf{a}\right)(t) & =\frac{1}{2 \pi} \sum_{l=1}^{s} \int_{-M_{l}-N_{l}}^{-M_{l}} \int_{-\infty}^{\infty}(\vec{a}(u))^{\top} F(\lambda)(F(\lambda)+G(\lambda))^{-1} G(\lambda) e^{i \lambda(u-t)} d \lambda d u \\
\vec{a}(t) & \in L_{2}(S), \quad t \in S .
\end{aligned}
$$

Making use of the introduced operators, relation (6) can be represented in the form

$$
\left(\mathbf{R}_{s} \mathbf{a}\right)(t)=\left(\mathbf{B}_{s} \mathbf{c}\right)(t), \quad t \in S
$$

Suppose that the operator $\mathbf{B}_{s}$ is invertible (see the paper by Salehi [38] for more details). Then the function $\vec{c}(t)$ can be calculated by the formula

$$
\vec{c}(t)=\left(\mathbf{B}_{s}^{-1} \mathbf{R}_{s} \mathbf{a}\right)(t), \quad t \in S .
$$

Consequently, the spectral characteristic $h(\lambda)$ of the estimate $\hat{A}_{s} \vec{\xi}$ is calculated by the formula

$$
\begin{aligned}
& (h(\lambda))^{\top}=\left(A_{s}(\lambda)\right)^{\top} F(\lambda)(F(\lambda)+G(\lambda))^{-1}-\left(C_{s}(\lambda)\right)^{\top}(F(\lambda)+G(\lambda))^{-1} \\
& C_{s}(\lambda)=\sum_{l=1}^{s} \int_{-M_{l}-N_{l}}^{-M_{l}}\left(\mathbf{B}_{s}^{-1} \mathbf{R}_{s} \mathbf{a}\right)(t) e^{i t \lambda} d t, \quad t \in S .
\end{aligned}
$$

The mean-square error of the estimate $\hat{A}_{s} \vec{\xi}$ can be calculated by the formula

$$
\Delta(h ; F, G)=\frac{1}{2 \pi} \int_{-\infty}^{\infty}\left(\left(A_{s}(\lambda)\right)^{\top} G(\lambda)+\left(C_{s}(\lambda)\right)^{\top}\right)(F(\lambda)+G(\lambda))^{-1} F(\lambda) \times
$$




$$
\begin{gathered}
\times(F(\lambda)+G(\lambda))^{-1}\left(\left(A_{s}(\lambda)\right)^{\top} G(\lambda)+\left(C_{s}(\lambda)\right)^{\top}\right)^{*} d \lambda+ \\
+\frac{1}{2 \pi} \int_{-\infty}^{\infty}\left(\left(A_{s}(\lambda)\right)^{\top} F(\lambda)-\left(C_{s}(\lambda)\right)^{\top}\right)(F(\lambda)+G(\lambda))^{-1} G(\lambda) \times \\
\times(F(\lambda)+G(\lambda))^{-1}\left(\left(A_{s}(\lambda)\right)^{\top} G(\lambda)+\left(C_{s}(\lambda)\right)^{\top}\right)^{*} d \lambda= \\
=\left\langle\left(\mathbf{R}_{s} \mathbf{a}\right)(t),\left(\mathbf{B}_{s}^{-1} \mathbf{R}_{s} \mathbf{a}\right)(t)\right\rangle+\left\langle\left(\mathbf{Q}_{s} \mathbf{a}\right)(t), \vec{a}(t)\right\rangle,
\end{gathered}
$$

where $\langle a(t), b(t)\rangle$ is the inner product in the space $L_{2}(S)$.

Let us summarize the obtained results and present them in the form of a theorem.

Theorem 2.1

Let $\{\vec{\xi}(t), t \in \mathbb{R}\}$ and $\{\vec{\eta}(t), t \in \mathbb{R}\}$ be uncorrelated multidimensional stationary stochastic processes with the spectral density matrices $F(\lambda)$ and $G(\lambda)$ which satisfy the minimality condition (1). Suppose that condition (3) is satisfied and the operator $\mathbf{B}_{s}$ is invertible. The spectral characteristic $h(\lambda)$ and the mean-square error $\Delta(F, G)$ of the optimal linear estimate of the functional $A_{s} \vec{\xi}$ which depends on the unknown values of the process $\vec{\xi}(t)$ based on observations of the process $\vec{\xi}(t)+\vec{\eta}(t)$ at points $t \in \mathbb{R} \backslash S$ can be calculated by formulas (8), (9).

Consider the case where the stationary process $\vec{\xi}(t)$ is observed without noise. In this case the spectral characteristic of the estimate $\hat{A}_{s} \vec{\xi}$ is of the form

$$
\begin{aligned}
& (h(\lambda))^{\top}=\left(A_{s}(\lambda)\right)^{\top}-\left(C_{s}(\lambda)\right)^{\top}(F(\lambda))^{-1}, \\
& C_{s}(\lambda)=\sum_{l=1}^{s} \int_{-M_{l}-N_{l}}^{-M_{l}} \vec{c}(t) e^{i t \lambda} d t,
\end{aligned}
$$

the relation (7) is of the form

$$
\vec{a}(t)=\left(\mathbf{B}_{s} \mathbf{c}\right)(t), \quad t \in S .
$$

Suppose that the operator $\mathbf{B}_{s}$ is invertible. Then the unknown function $\vec{c}(t)$ can be calculated by the formula

$$
\vec{c}(t)=\left(\mathbf{B}_{s}^{-1} \mathbf{a}\right)(t), \quad t \in S,
$$

and the spectral characteristic of the estimate $\hat{A}_{s} \vec{\xi}$ is of the form

$$
\begin{aligned}
& (h(\lambda))^{\top}=\left(A_{s}(\lambda)\right)^{\top}-\left(C_{s}(\lambda)\right)^{\top}(F(\lambda))^{-1}, \\
& C_{s}(\lambda)=\sum_{l=1}^{s} \int_{-M_{l}-N_{l}}^{-M_{l}}\left(\mathbf{B}_{s}^{-1} \mathbf{a}\right)(t) e^{i t \lambda} d t, \quad t \in S .
\end{aligned}
$$

The mean-square error of the estimate can be calculated by the formula

$$
\Delta(h ; F)=\left\langle\left(\mathbf{B}_{s}^{-1}\right) \mathbf{a}(t), \vec{a}(t)\right\rangle .
$$

The following theorem holds true.

\section{Theorem 2.2}

Let $\{\vec{\xi}(t), t \in \mathbb{R}\}$ be a multidimensional stationary stochastic process with the spectral density matrix $F(\lambda)$, which satisfies the minimality condition

$$
\int_{-\infty}^{\infty}(b(\lambda))^{\top}(F(\lambda))^{-1} \overline{b(\lambda)} d \lambda<\infty
$$


for a nonzero vector-valued function of the exponential type $b(\lambda)=\sum_{l=1}^{s} \int_{-M_{l}-N_{l}}^{-M_{l}} \vec{\alpha}(t) e^{i t \lambda} d t$. Suppose that condition (3) is satisfied and the operator $\mathbf{B}_{s}$ is invertible. The spectral characteristic $h(\lambda)$ and the mean-square error $\Delta(F)$ of the optimal linear estimate of the functional $A_{s} \vec{\xi}$ which depends on the unknown values of the process $\vec{\xi}(t)$ based on observations of the process $\vec{\xi}(t)$ at points $t \in \mathbb{R} \backslash S, S=\bigcup_{l=1}^{s}\left[-M_{l}-N_{l},-M_{l}\right]$, can be calculated by formulas (12), (13).

\section{Minimax approach to interpolation problem for stationary processes with missing observations}

The derived formulas can be applied only in the case of spectral certainty, where the spectral density matrices of the considered processes are exactly known. However, in practice, we do not have exact values of the spectral density matrices while, instead, we have a class of admissible spectral density matrices, where the spectral density matrices of the processes belong to. In this case, the minimax method can be applied to estimate the value of the functional. This method allows us to find estimates that minimize the maximum values of the mean-square errors of the estimates for all spectral density matrices from a given class of admissible spectral density matrices. For description of the minimax method we introduce the following definitions (see Moklyachuk [23, 24], and Moklyachuk and Masytka [28]).

Definition 3.1. For a given class of spectral densities $D=D_{F} \times D_{G}$ the spectral densities $F^{0}(\lambda) \in D_{F}, G^{0}(\lambda) \in$ $D_{G}$ are called the least favorable in the class $D$ for the optimal linear interpolation of the functional $A_{s} \vec{\xi}$ if the following relation holds true

$$
\Delta\left(F^{0}, G^{0}\right)=\Delta\left(h\left(F^{0}, G^{0}\right) ; F^{0}, G^{0}\right)=\max _{(F, G) \in D_{F} \times D_{G}} \Delta(h(F, G) ; F, G) .
$$

Definition 3.2. For a given class of spectral densities $D=D_{F} \times D_{G}$ the spectral characteristic $h^{0}(\lambda)$ of the optimal linear interpolation of the functional $A_{s} \vec{\xi}$ is called minimax-robust if there are satisfied conditions

$$
\begin{gathered}
h^{0}(\lambda) \in H_{D}=\bigcap_{(F, G) \in D_{F} \times D_{G}} L_{2}^{s}(F+G), \\
\min _{h \in H_{D}} \max _{(F, G) \in D} \Delta(h ; F, G)=\max _{(F, G) \in D} \Delta\left(h^{0} ; F, G\right) .
\end{gathered}
$$

From the introduced definitions and formulas derived in previous section we can obtain the following statements.

Lemma 3.1

The spectral densities $F_{0}(\lambda) \in D_{F}, G_{0}(\lambda) \in D_{G}$ satisfying the minimality condition (1) are the least favorable in the class $D=D_{F} \times D_{G}$ for the optimal linear interpolation of the functional $A_{s} \vec{\xi}$, if the Fourier coefficients of the functions

$$
\left(F^{0}(\lambda)+G^{0}(\lambda)\right)^{-1}, \quad F^{0}(\lambda)\left(F^{0}(\lambda)+G^{0}(\lambda)\right)^{-1}, \quad F^{0}(\lambda)\left(F^{0}(\lambda)+G^{0}(\lambda)\right)^{-1} G^{0}(\lambda)
$$

define operators $\mathbf{B}_{s}^{0}, \mathbf{R}_{s}^{0}, \mathbf{Q}_{s}^{0}$, which determine a solution of the constrained optimization problem

$$
\begin{aligned}
& \max _{(F, G) \in D_{F} \times D_{G}}\left\langle\left(\mathbf{R}_{s} \mathbf{a}\right)(t),\left(\mathbf{B}_{s}^{-1} \mathbf{R}_{s} \mathbf{a}\right)(t)\right\rangle+\left\langle\left(\mathbf{Q}_{s} \mathbf{a}\right)(t), \vec{a}(t)\right\rangle= \\
& =\left\langle\left(\mathbf{R}_{s}^{0} \mathbf{a}\right)(t),\left(\left(\mathbf{B}_{s}^{0}\right)^{-1} \mathbf{R}_{s}^{0} \mathbf{a}\right)(t)\right\rangle+\left\langle\left(\mathbf{Q}_{s}^{0} \mathbf{a}\right)(t), \vec{a}(t)\right\rangle .
\end{aligned}
$$

The minimax spectral characteristic $h^{0}=h\left(F^{0}, G^{0}\right)$ is determined by the formula (8) if $h\left(F^{0}, G^{0}\right) \in H_{D}$. 
Corrolary 3.1

Let the spectral density $F^{0}(\lambda) \in D_{F}$ satisfy the minimality condition (14). The spectral density $F^{0}(\lambda) \in D_{F}$ is the least favorable in the class $D_{F}$ for the optimal linear interpolation of the functional $A_{s} \vec{\xi}$ from observations of the process $\vec{\xi}(t)$ at points $t \in \mathbb{R} \backslash S$ if the Fourier coefficients of the function $\left(F^{0}(\lambda)\right)^{-1}$ define the operator $\mathbf{B}_{s}^{0}$ which determines a solution of the constrained optimization problem

$$
\max _{F \in D_{F}}\left\langle\left(\mathbf{B}_{s}^{-1} \mathbf{a}\right)(t), \vec{a}(t)\right\rangle=\left\langle\left(\left(\mathbf{B}_{s}^{0}\right)^{-1} \mathbf{a}\right)(t), \vec{a}(t)\right\rangle .
$$

The minimax spectral characteristic $h^{0}=h\left(F^{0}\right)$ is determined by the formula (12) if $h\left(F^{0}\right) \in H_{D_{F}}$.

For more detailed analysis of properties of the least favorable spectral densities and the minimax-robust spectral characteristics we observe that the least favorable spectral densities $F^{0}(\lambda), G^{0}(\lambda)$ and the minimax spectral characteristic $h^{0}=h\left(F^{0}, G^{0}\right)$ form a saddle point of the function $\Delta(h ; F, G)$ on the set $H_{D} \times D$. The saddle point inequalities

$$
\Delta\left(h^{0} ; F, G\right) \leq \Delta\left(h^{0} ; F^{0}, G^{0}\right) \leq \Delta\left(h ; F^{0}, G^{0}\right), \quad \forall h \in H_{D}, \forall F \in D_{F}, \forall G \in D_{G},
$$

hold true if $h^{0}=h\left(F^{0}, G^{0}\right)$ and $h\left(F^{0}, G^{0}\right) \in H_{D}$, where $\left(F^{0}, G^{0}\right)$ is a solution to the constrained optimization problem

$$
\sup _{(F, G) \in D_{F} \times D_{G}} \Delta\left(h\left(F^{0}, G^{0}\right) ; F, G\right)=\Delta\left(h\left(F^{0}, G^{0}\right) ; F^{0}, G^{0}\right) .
$$

The linear functional $\Delta\left(h\left(F^{0}, G^{0}\right) ; F, G\right)$ is calculated by the formula

$$
\begin{gathered}
\Delta\left(h\left(F^{0}, G^{0}\right) ; F, G\right)= \\
=\frac{1}{2 \pi} \int_{-\infty}^{\infty}\left(\left(A_{s}(\lambda)\right)^{\top} G^{0}(\lambda)+\left(C_{s}^{0}(\lambda)\right)^{\top}\right)\left(F^{0}(\lambda)+G^{0}(\lambda)\right)^{-1} F(\lambda) \times \\
\times\left(F^{0}(\lambda)+G^{0}(\lambda)\right)^{-1}\left(\left(A_{s}(\lambda)\right)^{\top} G^{0}(\lambda)+\left(C_{s}^{0}(\lambda)\right)^{\top}\right)^{*} d \lambda+ \\
+\frac{1}{2 \pi} \int_{-\infty}^{\infty}\left(\left(A_{s}(\lambda)\right)^{\top} F^{0}(\lambda)-\left(C_{s}^{0}(\lambda)\right)^{\top}\right)\left(F^{0}(\lambda)+G^{0}(\lambda)\right)^{-1} G(\lambda) \times \\
\times\left(F^{0}(\lambda)+G^{0}(\lambda)\right)^{-1}\left(\left(A_{s}(\lambda)\right)^{\top} G^{0}(\lambda)-\left(C_{s}^{0}(\lambda)\right)^{\top}\right)^{*} d \lambda, \\
C_{s}^{0}(\lambda)=\sum_{l=1}^{s} \int_{-M_{l}-N_{l}}^{-M_{l}}\left(\left(\mathbf{B}_{s}^{0}\right)^{-1} \mathbf{R}_{s}^{0} \mathbf{a}\right)(t) e^{i t \lambda} d t, \quad t \in S .
\end{gathered}
$$

The constrained optimization problem (17) is equivalent to the unconstrained optimization problem (see Pshenichnyj [35])

$$
\Delta_{D}(F, G)=-\Delta\left(h\left(F^{0}, G^{0}\right) ; F, G\right)+\delta\left((F, G) \mid D_{F} \times D_{G}\right) \rightarrow \mathrm{inf},
$$

where $\delta\left((F, G) \mid D_{F} \times D_{G}\right)$ is the indicator function of the set $D=D_{F} \times D_{G}$. A solution of the problem (18) is characterized by the condition $0 \in \partial \Delta_{D}\left(F^{0}, G^{0}\right)$, where $\partial \Delta_{D}\left(F^{0}, G^{0}\right)$ is the subdifferential of the convex functional $\Delta_{D}(F, G)$ at point $\left(F^{0}, G^{0}\right)$, which is the necessary and sufficient condition under which the pair $\left(F^{0}, G^{0}\right)$ belongs to the set of minimums of the convex functional $\Delta\left(h\left(F^{0}, G^{0}\right) ; F, G\right)$. This condition makes it possible to find the least favourable spectral densities in some special classes of spectral densities $D$ (see books by Ioffe and Tihomirov [13], Pshenichnyj [35], Rockafellar [36]).

Note, that the form of the functional $\Delta\left(h\left(F^{0}, G^{0}\right) ; F, G\right)$ is convenient for application of the Lagrange method of indefinite multipliers for finding solution to the problem (18). Making use of the method of Lagrange multipliers and the form of subdifferentials of the indicator functions we describe relations that determine the least favourable spectral densities in some special classes of spectral densities (see books by Moklyachuk [22, 23], Moklyachuk and Masyutka [28] for additional details).

Taking into consideration the introduced definitions and the derived relations we can verify that the following lemma holds true. 
Lemma 3.2

Let $\left(F^{0}, G^{0}\right)$ be a solution of the optimization problem (18). The spectral densities $F^{0}(\lambda), G^{0}(\lambda)$ are the least favorable in the class $D=D_{F} \times D_{G}$ and the spectral characteristic $h^{0}=h\left(F^{0}, G^{0}\right)$ is the minimax of the optimal linear estimate of the functional $A_{s} \vec{\xi}$ if $h\left(F^{0}, G^{0}\right) \in H_{D}$.

\section{Least favorable spectral densities in the class $D=D_{0} \times D_{\varepsilon}$}

Consider the problem of mean-square optimal interpolation of the functional $A_{s} \vec{\xi}$ in the case where the spectral density matrices of the processes are not exactly known while the admissible spectral density matrices are from the class $D=D_{0} \times D_{\varepsilon}$, where

$$
\begin{gathered}
D_{0}^{1}=\left\{F(\lambda) \mid \frac{1}{2 \pi} \int_{-\infty}^{\infty} \operatorname{Tr} F(\lambda) d \lambda=p\right\} \\
D_{\varepsilon}^{1}=\left\{G(\lambda) \mid \operatorname{Tr} G(\lambda)=(1-\varepsilon) \operatorname{Tr} G_{1}(\lambda)+\varepsilon \operatorname{Tr} W(\lambda), \frac{1}{2 \pi} \int_{-\infty}^{\infty} \operatorname{Tr} G(\lambda) d \lambda=q\right\} ; \\
D_{0}^{2}=\left\{F(\lambda) \mid \frac{1}{2 \pi} \int_{-\infty}^{\infty} f_{k k}(\lambda) d \lambda=p_{k}, k=\overline{1, T}\right\}, \\
D_{\varepsilon}^{2}=\left\{G(\lambda) \mid g_{k k}(\lambda)=(1-\varepsilon) g_{k k}^{1}(\lambda)+\varepsilon w_{k k}(\lambda), \frac{1}{2 \pi} \int_{-\infty}^{\infty} g_{k k}(\lambda) d \lambda=q_{k}, k=\overline{1, T}\right\} ; \\
D_{\varepsilon}^{3}=\left\{G(\lambda) \mid\left\langle\frac{1}{2 \pi} \int_{-\infty}^{\infty}\left\langle B_{1}, F(\lambda)\right\rangle d \lambda=p\right\},\right. \\
D_{0}^{4}=\left\{F(\lambda) \mid \frac{1}{2 \pi} \int_{-\infty}^{\infty} F(\lambda) d \lambda=P\right\}, \\
D_{\varepsilon}^{4}=\left\{G(\lambda) \mid G(\lambda)=(1-\varepsilon) G_{1}(\lambda)+\varepsilon W(\lambda), \frac{1}{2 \pi} \int_{-\infty}^{\infty} G(\lambda) d \lambda=Q\right\} .
\end{gathered}
$$

Here $G_{1}(\lambda)$ is a known and fixed spectral density matrix while $W(\lambda)$ is an unknown spectral density matrix, $p, q, p_{k}, q_{k}, k=\overline{1, T}$ are fixed numbers, $P, Q, B_{1}, B_{2}$ are fixed positive definite Hermitian matrices. The class $D_{\varepsilon}$ describes " $\varepsilon$-contamination" model of stochastic processes.

From the condition $0 \in \partial \Delta_{D}\left(F^{0}, G^{0}\right)$ we find the following equations which determine the least favourable spectral densities for these sets of admissible spectral densities.

For the first pair $D_{0}^{1} \times D_{\varepsilon}^{1}$ we have equations

$$
\begin{gathered}
\left(\left(A_{s}(\lambda)\right)^{\top} G^{0}(\lambda)+\left(C_{s}^{0}(\lambda)\right)^{\top}\right)^{*}\left(\left(A_{s}(\lambda)\right)^{\top} G^{0}(\lambda)+\left(C_{s}^{0}(\lambda)\right)^{\top}\right)=\alpha^{2}\left(F^{0}(\lambda)+G^{0}(\lambda)\right)^{2}, \\
\left(\left(A_{s}(\lambda)\right)^{\top} F^{0}(\lambda)-\left(C_{s}^{0}(\lambda)\right)^{\top}\right)^{*}\left(\left(A_{s}(\lambda)\right)^{\top} F^{0}(\lambda)-\left(C_{s}^{0}(\lambda)\right)^{\top}\right)=\left(\beta^{2}+\gamma(\lambda)\right)\left(F^{0}(\lambda)+G^{0}(\lambda)\right)^{2},
\end{gathered}
$$

where $\alpha^{2}, \beta^{2}$ are Lagrange multipliers, $\gamma(\lambda) \leq 0$ and $\gamma(\lambda)=0$ if $\operatorname{Tr} F^{0}(\lambda)>(1-\varepsilon) \operatorname{Tr} G_{1}(\lambda)$.

For the second pair $D_{0}^{2} \times D_{\varepsilon}^{2}$ we have equations

$$
\begin{gathered}
\left(\left(A_{s}(\lambda)\right)^{\top} G^{0}(\lambda)+\left(C_{s}^{0}(\lambda)\right)^{\top}\right)^{*}\left(\left(A_{s}(\lambda)\right)^{\top} G^{0}(\lambda)+\left(C_{s}^{0}(\lambda)\right)^{\top}\right)= \\
=\left(F^{0}(\lambda)+G^{0}(\lambda)\right)\left\{\alpha_{k}^{2} \delta_{k l}\right\}_{k, l=1}^{T}\left(F^{0}(\lambda)+G^{0}(\lambda)\right), \\
\left(\left(A_{s}(\lambda)\right)^{\top} F^{0}(\lambda)-\left(C_{s}^{0}(\lambda)\right)^{\top}\right)^{*}\left(\left(A_{s}(\lambda)\right)^{\top} F^{0}(\lambda)-\left(C_{s}^{0}(\lambda)\right)^{\top}\right)= \\
=\left(F^{0}(\lambda)+G^{0}(\lambda)\right)\left\{\left(\beta_{k}^{2}+\gamma_{k}(\lambda)\right) \delta_{k l}\right\}_{k, l=1}^{T}\left(F^{0}(\lambda)+G^{0}(\lambda)\right),
\end{gathered}
$$


where $\alpha_{k}^{2}, \beta_{k}^{2}$ are Lagrange multipliers, $\delta_{k l}$ are Kronecker symbols, $\gamma_{k}(\lambda) \leq 0$ and $\gamma_{k}(\lambda)=0$ if $g_{k k}^{0}(\lambda)>(1-$ $\varepsilon) g_{k k}^{1}(\lambda)$.

For the third pair $D_{0}^{3} \times D_{\varepsilon}^{3}$ we have equations

$$
\begin{gathered}
\left(\left(A_{s}(\lambda)\right)^{\top} G^{0}(\lambda)+\left(C_{s}^{0}(\lambda)\right)^{\top}\right)^{*}\left(\left(A_{s}(\lambda)\right)^{\top} G^{0}(\lambda)+\left(C_{s}^{0}(\lambda)\right)^{\top}\right)= \\
=\alpha^{2}\left(F^{0}(\lambda)+G^{0}(\lambda)\right) B_{1}^{\top}\left(F^{0}(\lambda)+G^{0}(\lambda)\right), \\
\left(\left(A_{s}(\lambda)\right)^{\top} F^{0}(\lambda)-\left(C_{s}^{0}(\lambda)\right)^{\top}\right)^{*}\left(\left(A_{s}(\lambda)\right)^{\top} F^{0}(\lambda)-\left(C_{s}^{0}(\lambda)\right)^{\top}\right)= \\
=\left(\beta^{2}+\gamma^{\prime}(\lambda)\right)\left(F^{0}(\lambda)+G^{0}(\lambda)\right) B_{2}^{\top}\left(F^{0}(\lambda)+G^{0}(\lambda)\right),
\end{gathered}
$$

where $\alpha^{2}, \beta^{2}$ are Lagrange multipliers, $\gamma^{\prime}(\lambda) \leq 0$ and $\gamma^{\prime}(\lambda)=0$ if $\left\langle B_{2}, G^{0}(\lambda)\right\rangle>(1-\varepsilon)\left\langle B_{2}, G_{1}(\lambda)\right\rangle$.

For the fourth pair $D_{0}^{4} \times D_{\varepsilon}^{4}$ we have equations

$$
\begin{gathered}
\left(\left(A_{s}(\lambda)\right)^{\top} G^{0}(\lambda)+\left(C_{s}^{0}(\lambda)\right)^{\top}\right)^{*}\left(\left(A_{s}(\lambda)\right)^{\top} G^{0}(\lambda)+\left(C_{s}^{0}(\lambda)\right)^{\top}\right)= \\
=\left(F^{0}(\lambda)+G^{0}(\lambda)\right) \vec{\alpha} \cdot \vec{\alpha}^{*}\left(F^{0}(\lambda)+G^{0}(\lambda)\right), \\
\left(\left(A_{s}(\lambda)\right)^{\top} F^{0}(\lambda)-\left(C_{s}^{0}(\lambda)\right)^{\top}\right)^{*}\left(\left(A_{s}(\lambda)\right)^{\top} F^{0}(\lambda)-\left(C_{s}^{0}(\lambda)\right)^{\top}\right)= \\
=\left(F^{0}(\lambda)+G^{0}(\lambda)\right)\left(\vec{\beta} \cdot \vec{\beta}^{*}+\Gamma(\lambda)\right)\left(F^{0}(\lambda)+G^{0}(\lambda)\right),
\end{gathered}
$$

where $\vec{\alpha}, \vec{\beta}$ are Lagrange multipliers, $\Gamma(\lambda) \leq 0$ and $\Gamma_{3}(\lambda)=0$ if $G^{0}(\lambda)>(1-\varepsilon) G_{1}(\lambda)$.

Thus, the following statement holds true.

\section{Theorem 4.1}

The least favorable spectral densities $F^{0}(\lambda), G^{0}(\lambda)$ in the classes $D_{0} \times D_{\varepsilon}$ for the optimal linear interpolation of the functional $A_{s} \vec{\xi}$ are determined by relations (19), (20) for the first pair $D_{0}^{1} \times D_{\varepsilon}^{1}$ of sets of admissible spectral densities; (21), (22) for the second pair $D_{0}^{2} \times D_{\varepsilon}^{2}$ of sets of admissible spectral densities; (23), (24) for the third pair $D_{0}^{3} \times D_{\varepsilon}^{3}$ of sets of admissible spectral densities; (25), (26) for the fourth pair $D_{0}^{4} \times D_{\varepsilon}^{4}$ of sets of admissible spectral densities; the minimality condition (1); the constrained optimization problem (15) and restrictions on densities from the corresponding classes $D_{0} \times D_{\varepsilon}$. The minimax-robust spectral characteristic of the optimal estimate of the functional $A_{s} \vec{\xi}$ is determined by the formula (8).

\section{Corrolary 4.1}

The least favorable spectral densities $F^{0}(\lambda)$ in the classes $D_{0}^{k}, k=1,2,3,4$, for the optimal linear interpolation of the functional $A_{s} \vec{\xi}$, which depends on the unknown values of the process $\vec{\xi}(t)$ based on observations of the process $\vec{\xi}(t)$ at points $t \in \mathbb{R} \backslash S$, are determined by the following equations, respectively,

$$
\begin{gathered}
\left(\left(C_{s}^{0}(\lambda)\right)^{\top}\right)^{*} \cdot\left(C_{s}^{0}(\lambda)\right)^{\top}=\alpha^{2}\left(F^{0}(\lambda)\right)^{2}, \\
\left(\left(C_{s}^{0}(\lambda)\right)^{\top}\right)^{*} \cdot\left(C_{s}^{0}(\lambda)\right)^{\top}=F^{0}(\lambda)\left\{\alpha_{k}^{2} \delta_{k l}\right\}_{k, l=1}^{T} F^{0}(\lambda), \\
\left(\left(C_{s}^{0}(\lambda)\right)^{\top}\right)^{*} \cdot\left(C_{s}^{0}(\lambda)\right)^{\top}=\alpha^{2} F^{0}(\lambda)\left(B_{2}\right)^{\top} F^{0}(\lambda), \\
\left(\left(C_{s}^{0}(\lambda)\right)^{\top}\right)^{*} \cdot\left(C_{s}^{0}(\lambda)\right)^{\top}=F^{0}(\lambda) \vec{\alpha} \cdot \vec{\alpha}^{*} F^{0}(\lambda),
\end{gathered}
$$

the minimality condition (14); the constrained optimization problem (16) and restrictions on densities from the corresponding classes $D_{0}^{k}, k=1,2,3,4$. The minimax spectral characteristic of the optimal estimate of the functional $A_{s} \vec{\xi}$ is determined by the formula (12).

\section{Corrolary 4.2}

The least favorable spectral densities $F^{0}(\lambda)$ in the classes $D_{\varepsilon}^{k}, k=1,2,3,4$, for the optimal linear interpolation of the functional $A_{s} \vec{\xi}$, which depends on the unknown values of the process $\vec{\xi}(t)$ based on observations of the process $\vec{\xi}(t)$ at points $t \in \mathbb{R} \backslash S$, are determined by the following equations, respectively,

$$
\left(\left(C_{s}^{0}(\lambda)\right)^{\top}\right)^{*} \cdot\left(C_{s}^{0}(\lambda)\right)^{\top}=\left(\beta^{2}+\gamma(\lambda)\right)\left(F^{0}(\lambda)\right)^{2},
$$




$$
\begin{gathered}
\left(\left(C_{s}^{0}(\lambda)\right)^{\top}\right)^{*} \cdot\left(C_{s}^{0}(\lambda)\right)^{\top}=F^{0}(\lambda)\left\{\left(\beta_{k}^{2}+\gamma_{k}(\lambda)\right) \delta_{k l}\right\}_{k, l=1}^{T} F^{0}(\lambda), \\
\left(\left(C_{s}^{0}(\lambda)\right)^{\top}\right)^{*} \cdot\left(C_{s}^{0}(\lambda)\right)^{\top}=\left(\beta^{2}+\gamma^{\prime}(\lambda)\right) F^{0}(\lambda)\left(B_{2}\right)^{\top} F^{0}(\lambda), \\
\left(\left(C_{s}^{0}(\lambda)\right)^{\top}\right)^{*} \cdot\left(C_{s}^{0}(\lambda)\right)^{\top}=F^{0}(\lambda)\left(\vec{\beta} \cdot \vec{\beta}^{*}+\Gamma(\lambda)\right) F^{0}(\lambda),
\end{gathered}
$$

the minimality condition (14); the constrained optimization problem (16) and restrictions on densities from the corresponding classes $D_{\varepsilon}^{k}, k=1,2,3,4$. The minimax spectral characteristic of the optimal estimate of the functional $A_{s} \vec{\xi}$ is determined by the formula (12).

\section{Least favorable spectral densities in the class $D=D_{V}^{U} \times D_{2 \delta}$}

Consider the problem of the mean-square optimal interpolation of the functional $A_{s} \vec{\xi}$ in the case where the spectral density matrices of the processes are not exactly known while the admissible spectral density matrices are from the class $D=D_{V}^{U} \times D_{2 \delta}$, where

$$
\begin{gathered}
D_{V}^{U^{1}}=\left\{F(\lambda) \mid \operatorname{Tr} V(\lambda) \leq \operatorname{Tr} F(\lambda) \leq \operatorname{Tr} U(\lambda), \frac{1}{2 \pi} \int_{-\infty}^{\infty} \operatorname{Tr} F(\lambda) d \lambda=p\right\} \\
D_{2 \delta}^{1}=\left\{\left.G(\lambda)\left|\frac{1}{2 \pi} \int_{-\infty}^{\infty}\right| \operatorname{Tr}\left(G(\lambda)-G_{1}(\lambda)\right)\right|^{2} d \lambda \leq \delta\right\} ; \\
D_{V}^{U^{2}}=\left\{F(\lambda) \mid v_{k k}(\lambda) \leq f_{k k}(\lambda) \leq u_{k k}(\lambda), \frac{1}{2 \pi} \int_{-\infty}^{\infty} f_{k k}(\lambda) d \lambda=p_{k}, k=\overline{1, T}\right\}, \\
D_{V}^{U^{3}}=\left\{F(\lambda) \mid\left\langle B_{1}, V(\lambda)\right\rangle \leq\left\langle B_{1}, F(\lambda)\right\rangle \leq\left\langle B_{1}, U(\lambda)\right\rangle, \frac{1}{2 \pi} \int_{-\infty}^{\infty}\left\langle B_{1}, F(\lambda)\right\rangle d \lambda=p\right\}, \\
D_{2 \delta}^{3}=\left\{\left.G(\lambda)\left|\frac{1}{2 \pi} \int_{-\infty}^{\infty}\right|\left\langle B_{2}, G(\lambda)-G_{1}(\lambda)\right\rangle\right|^{2} d \lambda \leq \delta\right\} ; \\
D_{V}^{U^{4}}=\left\{F(\lambda) \mid V(\lambda) \leq F(\lambda) \leq U(\lambda), \frac{1}{2 \pi} \int_{-\infty}^{\infty} F(\lambda) d \lambda=P\right\} . \\
D_{2 \delta}^{4}=\left\{G(\lambda)\left|\frac{1}{2 \pi} \int_{-\infty}^{\infty}\right| g_{i j}(\lambda)-\left.g_{i j}^{1}(\lambda)\right|^{2} d \lambda \leq \delta_{i}^{j}, i, j=\overline{1, T}\right\}
\end{gathered}
$$

Here the spectral density matrices $V(\lambda), U(\lambda), G_{1}(\lambda)$ are known and fixed, $\delta, p, \delta_{k}, p_{k}, k=\overline{1, T}, \delta_{i}^{j}, i, j=\overline{1, T}$, are fixed numbers, $P, B_{1}, B_{2}$ are fixed positive-definite Hermitian matrices.

The class $D_{V}^{U}$ describes the "strip" model of stochastic processes while the class $D_{2 \delta}$ describes " $\delta$-neighborhood" model in the space $L_{2}$ of a fixed bounded spectral density $G_{1}(\lambda)$.

From the condition $0 \in \partial \Delta_{D}\left(F^{0}, G^{0}\right)$ we find the following equations which determine the least favourable spectral densities for these sets of admissible spectral densities.

For the first pair $D_{V}^{U^{1}} \times D_{2 \delta}^{1}$ we have equations

$$
\begin{gathered}
\left(\left(A_{s}(\lambda)\right)^{\top} G^{0}(\lambda)+\left(C_{s}^{0}(\lambda)\right)^{\top}\right)^{*}\left(\left(A_{s}(\lambda)\right)^{\top} G^{0}(\lambda)+\left(C_{s}^{0}(\lambda)\right)^{\top}\right)= \\
=\left(\alpha^{2}+\gamma_{1}(\lambda)+\gamma_{2}(\lambda)\right)\left(F^{0}(\lambda)+G^{0}(\lambda)\right)^{2}, \\
\left(\left(A_{s}(\lambda)\right)^{\top} F^{0}(\lambda)-\left(C_{s}^{0}(\lambda)\right)^{\top}\right)^{*}\left(\left(A_{s}(\lambda)\right)^{\top} F^{0}(\lambda)-\left(C_{s}^{0}(\lambda)\right)^{\top}\right)= \\
=\beta^{2} \operatorname{Tr}\left(G^{0}(\lambda)-G_{1}(\lambda)\right)\left(F^{0}(\lambda)+G^{0}(\lambda)\right)^{2},
\end{gathered}
$$




$$
\frac{1}{2 \pi} \int_{-\infty}^{\infty}\left|\operatorname{Tr}\left(G(\lambda)-G_{1}(\lambda)\right)\right|^{2} d \lambda=\delta
$$

where $\alpha^{2}, \beta^{2}$ are Lagrange multipliers, $\gamma_{1}(\lambda) \leq 0$ and $\gamma_{1}(\lambda)=0$ if $\operatorname{Tr} F^{0}(\lambda)>\operatorname{Tr} V(\lambda), \gamma_{2}(\lambda) \geq 0$ and $\gamma_{2}(\lambda)=0$ if $\operatorname{Tr} F^{0}(\lambda)<\operatorname{Tr} U(\lambda)$.

For the second pair $D_{V}^{U^{2}} \times D_{2 \delta}^{2}$ we have equations

$$
\begin{gathered}
\left(\left(A_{s}(\lambda)\right)^{\top} G^{0}(\lambda)+\left(C_{s}^{0}(\lambda)\right)^{\top}\right)^{*}\left(\left(A_{s}(\lambda)\right)^{\top} G^{0}(\lambda)+\left(C_{s}^{0}(\lambda)\right)^{\top}\right)= \\
=\left(F^{0}(\lambda)+G^{0}(\lambda)\right)\left\{\left(\alpha_{k}^{2}+\gamma_{1 k}(\lambda)+\gamma_{2 k}(\lambda)\right) \delta_{k l}\right\}_{k, l=1}^{T}\left(F^{0}(\lambda)+G^{0}(\lambda)\right), \\
\left(\left(A_{s}(\lambda)\right)^{\top} F^{0}(\lambda)-\left(C_{s}^{0}(\lambda)\right)^{\top}\right)^{*}\left(\left(A_{s}(\lambda)\right)^{\top} F^{0}(\lambda)-\left(C_{s}^{0}(\lambda)\right)^{\top}\right)= \\
=\left(F^{0}(\lambda)+G^{0}(\lambda)\right)\left\{\beta_{k}^{2}\left(g_{k k}^{0}(\lambda)-g_{k k}^{1}(\lambda)\right) \delta_{k l}\right\}_{k, l=1}^{T}\left(F^{0}(\lambda)+G^{0}(\lambda)\right), \\
\frac{1}{2 \pi} \int_{-\infty}^{\infty}\left|g_{k k}(\lambda)-g_{k k}^{1}(\lambda)\right|^{2} d \lambda=\delta_{k}, k=\overline{1, T},
\end{gathered}
$$

where $\alpha_{k}^{2}, \beta_{k}^{2}$ are Lagrange multipliers, $\gamma_{1 k}(\lambda) \leq 0$ and $\gamma_{1 k}(\lambda)=0$ if $f_{k k}^{0}(\lambda)>v_{k k}(\lambda), \gamma_{2 k}(\lambda) \geq 0$ and $\gamma_{2 k}(\lambda)=0$ if $f_{k k}^{0}(\lambda)<u_{k k}(\lambda)$.

For the third pair $D_{V}^{U^{3}} \times D_{2 \delta}^{3}$ we have equations

$$
\begin{gathered}
\left(\left(A_{s}(\lambda)\right)^{\top} G^{0}(\lambda)+\left(C_{s}^{0}(\lambda)\right)^{\top}\right)^{*}\left(\left(A_{s}(\lambda)\right)^{\top} G^{0}(\lambda)+\left(C_{s}^{0}(\lambda)\right)^{\top}\right)= \\
=\left(\alpha^{2}+\gamma_{1}^{\prime}(\lambda)+\gamma_{2}^{\prime}(\lambda)\right)\left(F^{0}(\lambda)+G^{0}(\lambda)\right)\left(B_{1}\right)^{\top}\left(F^{0}(\lambda)+G^{0}(\lambda)\right), \\
\left(\left(A_{s}(\lambda)\right)^{\top} F^{0}(\lambda)-\left(C_{s}^{0}(\lambda)\right)^{\top}\right)^{*}\left(\left(A_{s}(\lambda)\right)^{\top} F^{0}(\lambda)-\left(C_{s}^{0}(\lambda)\right)^{\top}\right)= \\
=\beta^{2}\left\langle B_{2}, G^{0}(\lambda)-G_{1}(\lambda)\right\rangle\left(F^{0}(\lambda)+G^{0}(\lambda)\right)^{2}, \\
\frac{1}{2 \pi} \int_{-\infty}^{\infty}\left|\left\langle B_{2}, G(\lambda)-G_{1}(\lambda)\right\rangle\right|^{2} d \lambda=\delta,
\end{gathered}
$$

where $\alpha^{2}, \beta^{2}$ are Lagrange multipliers, $\gamma_{1}^{\prime}(\lambda) \leq 0$ and $\gamma_{1}^{\prime}(\lambda)=0$ if $\left\langle B_{1}, F^{0}(\lambda\rangle>\left\langle B_{1}, V(\lambda)\right\rangle, \gamma_{2}^{\prime}(\lambda) \geq 0\right.$ and $\gamma_{2}^{\prime}(\lambda)=0$ if $\left\langle B_{1}, F^{0}(\lambda\rangle<\left\langle B_{1}, U(\lambda)\right\rangle\right.$.

For the fourth pair $D_{V}^{U^{4}} \times D_{2 \delta}^{4}$ we have equations

$$
\begin{gathered}
\left(\left(A_{s}(\lambda)\right)^{\top} G^{0}(\lambda)+\left(C_{s}^{0}(\lambda)\right)^{\top}\right)^{*}\left(\left(A_{s}(\lambda)\right)^{\top} G^{0}(\lambda)+\left(C_{s}^{0}(\lambda)\right)^{\top}\right)= \\
=\left(F^{0}(\lambda)+G^{0}(\lambda)\right)\left(\vec{\alpha} \cdot \vec{\alpha}^{*}+\Gamma_{1}(\lambda)+\Gamma_{2}(\lambda)\right)\left(F^{0}(\lambda)+G^{0}(\lambda)\right) \\
\left(\left(A_{s}(\lambda)\right)^{\top} F^{0}(\lambda)-\left(C_{s}^{0}(\lambda)\right)^{\top}\right)^{*}\left(\left(A_{s}(\lambda)\right)^{\top} F^{0}(\lambda)-\left(C_{s}^{0}(\lambda)\right)^{\top}\right)= \\
=\left(F^{0}(\lambda)+G^{0}(\lambda)\right)\left\{\beta_{i j}\left(g_{i j}^{0}(\lambda)-g_{i j}^{1}(\lambda)\right)\right\}_{i, j=1}^{T}\left(F^{0}(\lambda)+G^{0}(\lambda)\right), \\
\frac{1}{2 \pi} \int_{-\infty}^{\infty}\left|g_{i j}(\lambda)-g_{i j}^{1}(\lambda)\right|^{2} d \lambda=\delta_{i}^{j}, i, j=\overline{1, T} .
\end{gathered}
$$

where $\vec{\alpha}, \beta_{i j}$ are Lagrange multipliers, $\Gamma_{1}(\lambda) \leq 0$ and $\Gamma_{1}(\lambda)=0$ if $F^{0}(\lambda)>V(\lambda), \Gamma_{2}(\lambda) \geq 0$ and $\Gamma_{2}(\lambda)=0$ if $F^{0}(\lambda)<U(\lambda)$.

The following theorem and corollaries hold true.

Theorem 5.1

The least favorable spectral densities $F^{0}(\lambda), G^{0}(\lambda)$ in the classes $D=D_{V}^{U} \times D_{2 \delta}$ for the optimal linear interpolation of the functional $A_{s} \vec{\xi}$ are determined by relations (35) - (37) for the first pair $D_{V}^{U^{1}} \times D_{2 \delta}^{1}$ of sets of admissible spectral densities; (38) - (40) for the second pair $D_{V}^{U^{2}} \times D_{2 \delta}^{2}$ of sets of admissible spectral densities; (41) - (43) for the third pair $D_{V}^{U^{3}} \times D_{2 \delta}^{3}$ of sets of admissible spectral densities; (44) - (46) for the fourth pair $D_{V}^{U^{4}} \times D_{2 \delta}^{4}$ of sets of admissible spectral densities; the minimality condition (1); the constrained optimization problem (15) and restrictions on densities from the corresponding classes $D=D_{V}^{U} \times D_{2 \delta}$. The minimax-robust spectral characteristic of the optimal estimate of the functional $A_{s} \vec{\xi}$ is determined by the formula (8). 


\section{Corrolary 5.1}

The least favorable spectral densities $F^{0}(\lambda)$ in the classes $D_{V}^{U^{k}}, k=1,2,3,4$, for the optimal linear estimation of the functional $A_{s} \vec{\xi}$, which depends on the unknown values of the process $\vec{\xi}(t)$ based on observations of the process $\vec{\xi}(t)$ at points $t \in \mathbb{R} \backslash S$, are determined by the following equations, respectively,

$$
\begin{gathered}
\left(\left(C_{s}^{0}(\lambda)\right)^{\top}\right)^{*} \cdot\left(C_{s}^{0}(\lambda)\right)^{\top}=\left(\alpha^{2}+\gamma_{1}(\lambda)+\gamma_{2}(\lambda)\right)\left(F^{0}(\lambda)\right)^{2}, \\
\left(\left(C_{s}^{0}(\lambda)\right)^{\top}\right)^{*} \cdot\left(C_{s}^{0}(\lambda)\right)^{\top}=F^{0}(\lambda)\left\{\beta_{k}^{2}\left(g_{k k}^{0}(\lambda)-g_{k k}^{1}(\lambda)\right) \delta_{k l}\right\}_{k, l=1}^{T} F^{0}(\lambda), \\
\left(\left(C_{s}^{0}(\lambda)\right)^{\top}\right)^{*} \cdot\left(C_{s}^{0}(\lambda)\right)^{\top}=\left(\alpha^{2}+\gamma_{1}^{\prime}(\lambda)+\gamma_{2}^{\prime}(\lambda)\right) F^{0}(\lambda)\left(B_{1}\right)^{\top} F^{0}(\lambda), \\
\left(\left(C_{s}^{0}(\lambda)\right)^{\top}\right)^{*} \cdot\left(C_{s}^{0}(\lambda)\right)^{\top}=F^{0}(\lambda)\left(\vec{\alpha} \cdot \vec{\alpha}^{*}+\Gamma_{1}(\lambda)+\Gamma_{2}(\lambda)\right) F^{0}(\lambda),
\end{gathered}
$$

the minimality condition (1); the constrained optimization problem (16) and restrictions on densities from the corresponding classes $D_{V}^{U^{k}}, k=1,2,3,4$. The minimax spectral characteristic of the optimal estimate of the functional $A_{s} \vec{\xi}$ is determined by the formula (12).

\section{Corrolary 5.2}

The least favorable spectral densities $F^{0}(\lambda)$ in the classes $D_{2 \delta}^{k}, k=1,2,3,4$, for the optimal linear estimation of the functional $A_{s} \vec{\xi}$, which depends on the unknown values of the process $\vec{\xi}(t)$ based on observations of the process $\vec{\xi}(t)$ at points $t \in \mathbb{R} \backslash S$, are determined by the following equations, respectively,

$$
\begin{gathered}
\left(\left(C_{s}^{0}(\lambda)\right)^{\top}\right)^{*} \cdot\left(C_{s}^{0}(\lambda)\right)^{\top}=\beta^{2} \operatorname{Tr}\left(F^{0}(\lambda)-G_{1}(\lambda)\right)\left(F^{0}(\lambda)\right)^{2}, \\
\left(\left(C_{s}^{0}(\lambda)\right)^{\top}\right)^{*} \cdot\left(C_{s}^{0}(\lambda)\right)^{\top}=F^{0}(\lambda)\left\{\beta_{k}^{2}\left(f_{k k}^{0}(\lambda)-g_{k k}^{1}(\lambda)\right) \delta_{k l}\right\}_{k, l=1}^{T} F^{0}(\lambda), \\
\left(\left(C_{s}^{0}(\lambda)\right)^{\top}\right)^{*} \cdot\left(C_{s}^{0}(\lambda)\right)^{\top}=\beta^{2}\left\langle B_{2}, F^{0}(\lambda)-G_{1}(\lambda)\right\rangle\left(F^{0}(\lambda)\right)^{2}, \\
\left(\left(C_{s}^{0}(\lambda)\right)^{\top}\right)^{*} \cdot\left(C_{s}^{0}(\lambda)\right)^{\top}=F^{0}(\lambda)\left\{\beta_{i j}\left(f_{i j}^{0}(\lambda)-g_{i j}^{1}(\lambda)\right)\right\}_{i, j=1}^{T} F^{0}(\lambda)
\end{gathered}
$$

the minimality condition (1); the constrained optimization problem (16) and the following restrictions on densities from the corresponding classes $D_{2 \delta}^{k}, k=1,2,3,4$, respectively,

$$
\begin{gathered}
\frac{1}{2 \pi} \int_{-\infty}^{\infty}\left|\operatorname{Tr}\left(G(\lambda)-G_{1}(\lambda)\right)\right|^{2} d \lambda=\delta, \\
\frac{1}{2 \pi} \int_{-\infty}^{\infty}\left|g_{k k}(\lambda)-g_{k k}^{1}(\lambda)\right|^{2} d \lambda=\delta_{k}, k=\overline{1, T}, \\
\frac{1}{2 \pi} \int_{-\infty}^{\infty}\left|\left\langle B_{2}, G(\lambda)-G_{1}(\lambda)\right\rangle\right|^{2} d \lambda=\delta, \\
\frac{1}{2 \pi} \int_{-\infty}^{\infty}\left|g_{i j}(\lambda)-g_{i j}^{1}(\lambda)\right|^{2} d \lambda=\delta_{i}^{j}, i, j=\overline{1, T} .
\end{gathered}
$$

The minimax spectral characteristic of the optimal estimate of the functional $A_{s} \vec{\xi}$ is determined by the formula (12). 


\section{Conclusions}

In this article we propose methods of the mean-square optimal linear interpolation of functionals which depend on the unknown values of a multidimensional continuous time stationary stochastic process based on observations of the process with an additive stationary stochastic noise process. The case of spectral certainty as well as the case of spectral uncertainty are considered. Under the condition of spectral certainty, where the spectral density matrices of the stationary processes are exactly known, we derive formulas for calculating the spectral characteristic and the mean-square error of the optimal estimate of the functional. The corresponding results are derived in the case of observations of the process without noise. In the case of spectral uncertainty, where the spectral density matrices of the stationary processes are not exactly known while some sets of admissible spectral density matrices are given, we apply the minimax-robust method of estimation. This method allows us to find estimates that minimize the maximum values of the mean-square errors of the estimates for all spectral density matrices from a given class of admissible spectral density matrices and derive relations which determine the least favourable spectral density matrices. These least favourable spectral density matrices are solutions of the optimization problem (18) which is characterized by the condition $0 \in \partial \Delta_{D}\left(F^{0}, G^{0}\right)$, where $\partial \Delta_{D}\left(F^{0}, G^{0}\right)$ is the subdifferential of the convex functional $\Delta_{D}(F, G)$ at point $\left(F^{0}, G^{0}\right)$. The form of the functional $\Delta\left(h\left(F^{0}, G^{0}\right) ; F, G\right)$ is convenient for application of the Lagrange method of indefinite multipliers for finding solution to the problem (18). Making use of the method of Lagrange multipliers and the form of subdifferentials of the indicator functions we describe relations that determine the least favourable spectral densities in some special classes of spectral densities.

\section{REFERENCES}

1. P. Bondon, Influence of missing values on the prediction of a stationary time series, Journal of Time Series Analysis, vol. 26, no. 4, pp. 519-525, 2005.

2. P. Bondon, Prediction with incomplete past of a stationary process, Stochastic Process and their Applications, vol. 98, pp. 67-76, 2002.

3. G. E. P. Box, G. M. Jenkins, G. C. Reinsel, and G. M. Ljung, Time series analysis. Forecasting and control. 5 rd ed., Wiley, 2016.

4. P. J. Brockwell, and R. A. Davis, Time series: Theory and methods. 2nd ed.. New York: Springer, 1998.

5. R. Cheng, A. G. Miamee, and M. Pourahmadi, Some extremal problems in $L^{p}(w)$, Proceedings of the American Mathematical Society, vol. 126, pp. 2333-2340, 1998.

6. R. Cheng, and M. Pourahmadi, Prediction with incomplete past and interpolation of missing values, Statistics \& Probability Letters, vol. 33, pp. 341-346, 1996.

7. J. Franke, On the robust prediction and interpolation of time series in the presence of correlated noise. Journal of Time Series Analysis, vol. 5, no. 4, pp. 227-244, 1984

8. J. Franke, Minimax robust prediction of discrete time series, Z. Wahrscheinlichkeitstheor. Verw. Gebiete, vol. 68, pp. 337-364, 1985.

9. J. Franke, and H. V. Poor, Minimax-robust filtering and finite-length robust predictors, Robust and Nonlinear Time Series Analysis. Lecture Notes in Statistics, Springer-Verlag, vol. 26, pp. 87-126, 1984.

10. I. I. Gikhman, and A. V. Skorokhod, The theory of stochastic processes. I., Berlin: Springer, 2004.

11. U. Grenander, A prediction problem in game theory, Arkiv för Matematik, vol. 3, pp. 371-379, 1957.

12. E. J. Hannan, Multiple time series, Wiley Series in Probability and Mathematical Statistics. New York etc.: John Wiley \& Sons, Inc. XI, 1970

13. A. D. Ioffe, and V. M. Tihomirov, Theory of extremal problems, Studies in Mathematics and its Applications, Vol. 6. Amsterdam, New York, Oxford: North-Holland Publishing Company. XII, 1979.

14. K. Karhunen, Über lineare Methoden in der Wahrscheinlichkeitsrechnung, Annales Academiae Scientiarum Fennicae. Ser. A I, vol. 37, 1947.

15. Y. Kasahara, M. Pourahmadi, and A. Inoue, Duals of random vectors and processes with applications to prediction problems with missing values, Statistics \& Probability Letters, vol. 79, no. 14, pp. 1637-1646, 2009.

16. S. A. Kassam, and H. V. Poor, Robust techniques for signal processing: A survey, Proceedings of the IEEE, vol. 73, no. 3, pp. 433-481, 1985

17. A. N. Kolmogorov, Selected works by A. N. Kolmogorov. Vol. II: Probability theory and mathematical statistics. Ed. by A. N. Shiryayev, Mathematics and Its Applications. Soviet Series. 26. Dordrecht etc. Kluwer Academic Publishers, 1992.

18. M. M. Luz, and M. P. Moklyachuk, Minimax interpolation problem for stochastic processes with stationary increments, Statistics, Optimization \& Information Computing, vol. 3, no. 1, pp. 30-41, 2015.

19. M. M. Luz, and M. P. Moklyachuk, Minimax interpolation of sequences with stationary increments and cointegrated sequences, Modern Stochastics: Theory and Applications, vol. 3, no. 1. pp. 59-87, 2016.

20. M. M. Luz, and M. P. Moklyachuk, Estimates of functionals from processes with stationary increments and cointegrated sequences, Kyiv: NVP "Interservis", 2016. 
21. M. M. Luz, and M. P. Moklyachuk, Minimax interpolation of stochastic processes with stationary increments from observations with noise, Theory of Probability and Mathematical Statistics, vol. 94, pp. 121-135, 2017.

22. M. P. Moklyachuk, Nonsmooth analysis and optimization, Kyiv University, Kyiv, 2008.

23. M. P. Moklyachuk, Robust estimations of functionals of stochastic processes, Kyiv University, Kyiv, 2008.

24. M. P. Moklyachuk, Minimax-robust estimation problems for stationary stochastic sequences, Statistics, Optimization \& Information Computing, vol. 3, no. 4, pp. 348-419, 2015.

25. M. P. Moklyachuk, and I. I. Golichenko, Periodically correlated processes estimates, LAP Lambert Academic Publishing, 2016.

26. M. P. Moklyachuk, and O. Yu. Masyutka, Interpolation of multidimensional stationary sequences, Theory of Probability and Mathematical Statistics, vol. 73, pp. 121-135, 2006.

27. M. P. Moklyachuk, and O. Yu. Masyutka, Robust estimation problems for stochastic processes, Theory of Stochastic Processes, vol. 12, no. 3-4, pp. 88-113, 2006.

28. M. P. Moklyachuk, and O. Yu. Masyutka, Minimax-robust estimation technique for stationary stochastic processes, LAP Lambert Academic Publishing, 2012.

29. M. P. Moklyachuk, and M. I. Sidei, Interpolation of stationary sequences observed with the noise, Theory of Probability and Mathematical Statistics, vol. 93, pp. 143-156, 2015.

30. M. P. Moklyachuk, and M. I. Sidei, Interpolation problem for stationary sequences with missing observations, Statistics, Optimization \& Information Computing, vol. 3, no. 3, pp. 259-275, 2015.

31. M. P. Moklyachuk, and M. I. Sidei, Filtering problem for stationary sequences with missing observations. Statistics, Optimization \& Information Computing, vol. 4, no. 4, pp. 308-325 , 2016.

32. M. P. Moklyachuk, and M. I. Sidei, Extrapolation problem for stationary sequences with missing observations, Statistics, Optimization \& Information Computing, vol. 5, no. 3, pp. 212-233, 2017.

33. M. M. Pelagatti, Time series modelling with unobserved components, New York: CRC Press, 2015.

34. M. Pourahmadi, A. Inoue, and Y. Kasahara, A prediction problem in $L^{2}(w)$. Proceedings of the American Mathematical Society, vol. 135, No. 4, pp. 1233-1239, 2007.

35. B. N. Pshenichnyj, Necessary conditions of an extremum, Pure and Applied mathematics. 4. New York: Marcel Dekker, 1971.

36. R. T. Rockafellar, Convex Analysis, Princeton University Press, 1997.

37. Yu. A. Rozanov, Stationary stochastic processes, San Francisco-Cambridge-London-Amsterdam: Holden-Day, 1967.

38. H. Salehi, Algorithms for linear interpolator and interpolation error for minimal stationary stochastic processes, The Annals of Probability, Vol. 7, No. 5, pp. 840-846, 1979.

39. K. S. Vastola, and H. V. Poor, An analysis of the effects of spectral uncertainty on Wiener filtering, Automatica, vol. 28, pp. 289-293, 1983.

40. N. Wiener, Extrapolation, interpolation and smoothing of stationary time series. With engineering applications, The M. I. T. Press, Massachusetts Institute of Technology, Cambridge, Mass., 1966.

41. A. M. Yaglom, Correlation theory of stationary and related random functions. Vol. 1: Basic results, Springer Series in Statistics, Springer-Verlag, New York etc., 1987.

42. A. M. Yaglom, Correlation theory of stationary and related random functions. Vol. 2: Supplementary notes and references, Springer Series in Statistics, Springer-Verlag, New York etc., 1987. 